\title{
Ferrofluids and Magnetorheological Fluids
}

\author{
Ladislau Vékás ${ }^{1,2}$ \\ ${ }^{1}$ Laboratory of Magnetic Fluids, Center for Fundamental and Advanced Technical Research, \\ Romanian Academy-Timisoara Branch, Bd.Mihai Viteazul 24, Timisoara 300223, Romania \\ ${ }^{2}$ National Center for Engineering of Systems with Complex Fluids, University POLITEHNICA of \\ Timisoara, Bd. Mihai Viteazul 1, Timisoara 300222, Romania
}

vekas@acad-tim.tm.edu.ro

\begin{abstract}
Keywords: magnetically controllable fluids, ferrofluids, magnetic fluids, magnetorheological fluids, magnetic nanoparticles, magnetic particles, colloidal stability, sterical stabilization, electrostatical stabilization, particle agglomerates, sedimentation rate, rheological properties, magnetorheological effect, small angle neutron scattering, dynamical light scattering
\end{abstract}

Abstract. Composition, synthesis and structural properties of ferrofluids and magnetorheological fluids are reviewed and compared. The similarities and main differences between the two types of magnetically controllable fluids are outlined and exemplified in the paper. Chemical synthesis and structural characterization of magnetizable fluids for engineering and biomedical applications are thoroughly discussed.

\section{Introduction}

Ferrofluids (FFs) or magnetic (nano)fluids (M(N)Fs)- a category of smart fluids, in particular magnetically controllable nanofluids synthesized in the 1960's [1-4] - are rather attractive for a large variety of applications [5], which require simultaneously fluid and magnetic properties. Ferrofluids are ultrastable colloids of nanosized sub-domain magnetic dipolar particles in appropriate carrier liquids, actually an achievement of colloid science. Macroscopically, these fluids manifest themselves as magnetizable liquid media due to the "integration" in the structure of the carrier of sub-domain permanent magnetic dipolar particles.

Magnetorheological fluids (MR fluids, MRFs), a quite different type of magnetically controllable fluids, are suspensions of micrometer range ferro- or ferrimagnetic multi-domain particles in a liquid matrix [6, 7]. Introduced in the early 1940's, these fluids fully recovered the interest of researchers during the last two decades and now are the key components of many high-tech applications, especially of semi-active damping devices [8]. The basic phenomena in magnetorheology are related to the possibility to control the structure of the two-phase fluid by relatively moderate magnetic fields. The applied field polarizes the multi-domain magnetizable particles, induces their aggregation in large agglomerates and gives rise to a significant viscosity increase, up to about $10^{3}$ times. The magnetic moment of particles is induced by the applied field, consequently is vanishing at zero field and the suspension recovers its initial state, i.e. the process of effective viscosity change is reversible.

There are essential differences between ferrofluids and MR fluids. The size of magnetic particles in a ferrofluid is in the nanometer range $(3-15 \mathrm{~nm})$, while in a usual MR fluid is well above one micrometer $(1-20 \mu \mathrm{m})$. Consequently, the magnetic moment of micronic particles in MRFs is field induced and their Brownian motion is negligible, while in the case of ferrofluids the magnetic nanoparticles have permanent dipole moment and perform intense thermal motion. Particle aggregation processes are reversible and rather intense in MR fluids and are induced by the applied magnetic field, which is their key feature in developing field controlled flow behavior. Agglomerate formation in ferrofluids is limited due to thermal motion of nanoparticles and their steric and/or electrostatic stabilization in the carrier liquid, therefore the field induced changes in flow behavior usually are not significant. 


\section{Synthesis of ferrofluids}

At nanoscale, a specific difficulty associated with the preparation of magnetic fluids is that the nanoparticles have large surface area-to-volume ratios and thus tend to aggregate to reduce their surface energy. In particular, magnetic metal oxide surfaces have extremely high surface energies $(>100 \mathrm{dyn} / \mathrm{cm})$ that make the production of nanoparticles very challenging. In addition, magnetic dipole-dipole attractions between particles enhance the difficulties experienced in the production of ferrofluids. Long-range, attractive van der Waals and magnetic forces are ubiquitous and therefore must be balanced by Coulombic, steric or other interactions to control the colloidal stability of dispersed nanoparticle system, even in intense and strongly non-uniform magnetic field.

FFs are colloidal systems composed of isolated particles with nanometer-sized dimensions that are stabilized by surfactant molecules and dispersed in solvent media. In the ideal case, these noninteracting systems derive their unique magnetic properties mostly from the reduced size of the isolated nanoparticles, and contributions from interparticle interactions are negligible.

The synthesis of ferrofluids has two main steps [9]: (a) the preparation of nano-sized magnetic particles and (b) the subsequent stabilization/dispersion of the nanoparticles in various non-polar and polar carrier liquids.

Magnetic nanoparticles. The chemical coprecipitation method proved to be versatile for the preparation of magnetite, maghemite and substituted ferrites nanoparticles suitable for magnetic fluid preparation. In the case of magnetite, nanoparticles can be prepared from the coprecipitation of hydroxides from an aqueous solution of $\mathrm{Fe}^{3+}$ and $\mathrm{Fe}^{2+}$ in the mole ratio of approximatively 2:1 using a base [10]. The reaction is complex and involves the conversion of the hydroxide particles to magnetite. Particles produced may be in fact a mixture of magnetite and maghemite. Studies were performed concerning the effect of precipitation temperature on the nature and size of particles, the effect of heating the precipitate in the alkaline medium and the effect of addition of surfactant to the reaction medium $[11,12]$. An essential feature related to the synthesis of magnetite ferrofluids is to ensure the optimum temperature of $80-82^{\circ} \mathrm{C}[12,13,14]$ for co-precipitation and stabilization (usually with oleic acid) in order to obtain only magnetite nanoparticles with adequate chemisorbed stabilizing layer. Cobalt ferrite and $\gamma-\mathrm{Fe}_{2} \mathrm{O}_{3}$ nanoparticles can be prepared applying a similar procedure but using $\mathrm{NaOH}$ in excess, instead of $\mathrm{NH}_{4} \mathrm{OH}$. A different procedure was developed for the synthesis of cobalt ferrite, maghemite and magnetite nanoparticles using a microemulsion technique $[15,16]$, which allowed the fine control of particle size.

Thermal decomposition of organo-metallic compounds (e.g., iron or cobalt carbonyl) in organic solvents is an important strategy of making monodisperse iron/cobalt-containing nanoparticles and finally, high magnetization magnetic fluids. The composition, size and size distribution can be finely controlled by varying the reaction mixtures and synthesis conditions $[17,18,19]$. Various size-selective synthesis procedures were developed $[20,21,22]$ to obtain mono-disperse $\mathrm{MFe}_{2} \mathrm{O}_{4}$ nanoparticles with diameters smaller than $20 \mathrm{~nm}$ and having tight size distribution (sometimes less than $10 \%$ standard deviation).

Co nanoparticles for technical grade high magnetization magnetic fluids, e.g for ferrohydrostatic bearings, were prepared by thermolysis of $\mathrm{Co}_{2}(\mathrm{CO})_{8}$ in the presence of $\mathrm{Al}\left(\mathrm{C}_{8} \mathrm{H}_{17}\right)_{3}$ [23].

Preparation of iron/iron oxide core-shell nanostructures by a two-steps laser pyrolysis procedure applied to iron pentacarbonyl (vapors) gas mixture is described in [24, 25]. A careful in situ passivation process (mild surface oxidation) was performed in the second step on the primary collected nanoparticles to be used in ferrofluid preparation.

Ferrofluids with organic carriers. Colloidal stability of ferrofluids is ensured by sterical stabilization of magnetic nanoparticles in the carrier. The surfactant coating on magnetic nanoparticles prevents clustering due to steric repulsion. Stabilization-dispersion of nanoparticles in organic liquids using various chain length organic surface-active molecules (e.g., fatty acids, polymers)- steric stabilization- is dependent on the liquid carrier properties and it should ensure 
long-term colloidal stability of ferrofluids, even in an intense and strongly non-uniform magnetic field. This is a key feature of ferrofluid synthesis [1] and it has to take into account the specific conditions of the envisaged application.

Long-term colloidal stability of ferrofluids, especially at high volume fraction of magnetic nanoparticles, is a complex issue connected to the synthesis procedure followed, including the nature of surfactant(s) and carrier liquid used. The dimensionless magnetic interaction energy, $\lambda_{\text {int }}=\mu_{0} \mathrm{~m}^{2} /\left(2 \pi k_{B} T d_{m}{ }^{3}\right)$, which is half the ratio of the dipolar energy of two aligned magnetic dipoles at close contact to the thermal energy, should be kept below 1 to ensure highly stable magnetic fluids. During preparation repulsive forces due to coating of magnetic cores are introduced [1] to prevent irreversible aggregation of particles produced by attractive van der Waals and dipolar interactions. When the dipolar interactions are much stronger than the thermal energies, $\lambda_{\text {int }}>2$ [26], particle chains start growing and forming more complex structures, depending on the particle volume fraction, size distribution, temperature and magnetic field applied. Consequently, the colloidal stability of magnetic nanofluids is dependent on this interplay between isotropic van der Waals and anisotropic dipolar forces. An interesting feature of ferrofluid synthesis is that the relative strengths and ranges of various interaction potentials can be controlled by the diameter of magnetic cores and the thickness of the stabilizing layer [1]. In the case of weakly stabilized magnetic colloids at relatively high volume fraction when the contact distance between nanoparticles is short enough, due to the van der Waals interactions clusters are formed. These clusters exhibit large dipole moments compared to individual particles and favour development of larger structures and formation of chain-like agglomerates, especially under the influence of an applied field. Tailoring of ferrofluids for various applications has to take into account all these structural features.

E.g., ferrofluids for sealing applications have to be prepared in such a way to ensure high magnetization, low viscosity, low or very low vapour pressure and long-term colloidal stability in intense (magnetic induction 1-1.5 T) and strongly non-uniform magnetic field (field gradient $10^{8}$ $10^{9} \mathrm{~A} / \mathrm{m}^{2}$ ). These requirements are sometimes difficult to fulfil simultaneously and impose special conditions on the stabilization procedure applied in ferrofluid preparation, to avoid irreversible magnetic field induced structural processes. Details of the synthesis procedures of ferrofluids with non-polar and polar organic carriers are given in [12, 27, 28, 29, 30, 31].

According to the synthesis procedures mentioned above, transformer oil $(\mathrm{TR} 30)$, diesters $(\mathrm{DOA}=$ dioctyl adipate; $\mathrm{DOF}=$ dioctyl phtalate and DOS = dioctyl sebacate) and high vacuum oil (HVO (KW, Merck)) based ferrofluids were prepared for various type of magnetic fluid seals (MFSs) [31, 32], using fatty acids and polymers as primary and secondary surfactants.

$\mathrm{Co}, \mathrm{FeCo}$, and $\mathrm{Fe}$ nanoparticles synthesized by thermolysis $[33,34]$ of the carbonyl precursors in the presence of aluminium organics can be peptized after "smooth oxidation" yielding ferrofluids which were remarkably stable and which showed a high saturation magnetization and high volume concentrations (e.g. 10 vol.\% Co) [23]. Several surfactants such as KorantinSH (N-oleyl sarcosine, BASF AG), or AOT (sodium dioctyl sulfosuccinate, SERVA2), LP4 (fatty acid condensation polymer, ICI Ltd.) were applied to prepare ferrofluids in various organic media, e.g., kerosene, vacuum and mineral oil. Referring to magnetic fluids based on metallic $\mathrm{Co}, \mathrm{Fe} / \mathrm{Co}$, and $\mathrm{Fe}$ nanoparticles in various organic media [33], their saturation magnetization attains $190 \mathrm{kA} / \mathrm{m}$, the highest value reported up to now.

Water based ferrofluids. Electrostatic and steric stabilization procedures are both applicable for water carrier. Formation of similarly charged electric double layer [35, 36, 37] and/or of adsorption layers $[38,39,12,13,40,41,42,14,43,44]$ on the particle surface enhances the stability of magnetic colloids in aqueous medium.

The applications of magnetic fluids stabilized solely by electrostatic repulsion [35, 36] are somewhat restricted since this stabilizing mechanism is overly sensitive to conditions such as $\mathrm{pH}$ and ionic strength and offers little flexibility for tuning the surface properties of the particles. 
Double layer steric and electrostatic (combined) stabilization of magnetic nanoparticles in water carrier with dodecyl-benzensulphonic acid (DBSA+DBSA) double layer, proved to be efficient when colloidal stability had to be ensured up to relatively large values (over 0.10 ) of the particle volume fraction [28]. Improved colloidal stability in a wide range of $\mathrm{pH}$ and enhanced salt tolerance is expected for these nanofluids based on a manifold analysis of the mechanism of combined stabilization of magnetite particles in aqueous systems [45]. For most of biomedical uses the magnetic nanoparticles [46] should be below $15 \mathrm{~nm}$ in size and stably dispersed in water [47]. The attractive interactions between magnetic particles, when preponderant, may lead to various types of agglomerates, usually in the shape of linear chains quasi-parallel to the applied magnetic field or drop-like aggregates [48]. The ferrofluid becomes a biphasic system i.e. condensed-phase droplets form in equilibrium with the uncondensed phase matrix. The density of the condensed phase droplets increases with decreasing temperature and increasing magnetic field intensity. The kinetics of phase condensation was found to be influenced mainly by the temperature. The growth process of condensed-phase drops evolves in two stages: small primary agglomerates grow and stick together into large secondary drop-wise agglomerates. Particle condensation has to be impeded by advanced stabilization of water based ferrofluids for biomedical applications, because the agglomeration processes are much intensified under physiological conditions [50].

Biocompatible FFs were synthesized using LA, MA or OA as double layer surfactant [51, 52] instead of DBSA used only for technical grade FFs. Adsorption of fatty acids, the charge neutralization and overcharging due to the first and second layer formation, the effect of dilution, $\mathrm{pH}$ and salt concentration are the most important factors in case of FFs for biomedical application.

\section{Structure formation and colloidal stability}

DLS investigations. Colloidal stability of dilute water based ferrofluids prepared [51, 52], in particular $\mathrm{pH}$ dependence and salt tolerance were examined using DLS technique. DBS acid stabilized FF samples are not biocompatible and were considered for comparative investigations. Besides an attempt for the direct sizing of dense FFs with NanoZS apparatus (Malvern Co.) working in backscattering mode, the samples were characterized concerning the $\mathrm{pH}$-dependent stability and salt tolerance of these FFs in dilute aqueous systems.

The double DBSA layer stabilized FF was found to be strongly aggregated, the average size of hydrodynamic units varied between 160 and $270 \mathrm{~nm}$ depending on the $\mathrm{pH}$ from 3.4 to 7 and the salt concentration up to $0.15 \mathrm{M} \mathrm{NaCl}$. The combined layers of DBSA with LA and MA resulted also in relatively large aggregates $(\sim 64 \mathrm{~nm}$ at $\mathrm{pH} \sim 6.2)$ and $(\sim 33 \mathrm{~nm}$ at $\mathrm{pH} \sim 6.3)$ respectively, in accordance with SANS result [49]. Much better stabilization was reached with the LA, MA and OA double layers. It can be stated that the hydrophobic interactions are favored in the case of OA+OA and $\mathrm{MA}+\mathrm{MA}$ double layer stabilized ferrofluids, the magnetite nanoparticles with their coating are dispersed well, significant aggregation cannot be observed even in fairly dilute systems up to the physiological salt concentration in the favored $\mathrm{pH}$ region [52].

Small angle neutron scattering (SANS) is one of the most efficient methods of nanostructural investigation of ferrofluids [49]. The differential scattering cross-section per sample volume, the scattering intensity is quite sensitive to structural features of the studied system at the scale of 1$100 \mathrm{~nm}$. The sizes of magnetic particles in ferrofluids $(3-15 \mathrm{~nm})$ and the characteristic correlation length between particles are mostly in this dimensional range. SANS investigations including specific techniques (contrast variation, scattering of polarized neutrons SANSPOL) give information about particle structure (size, surfactant shell thickness, composition of core and shell, solvent rate of penetration in surfactant layer, micelles), magnetic structure (magnetic size and composition), particle interaction (interparticle potential, magnetic moment correlation, phase separation) and cluster formation (aggregation and chain formation). Major advantages of SANS in comparison with other structural methods are due to the possibilities of contrast variation [53] in the studied systems by substitution hydrogen/deuterium, as well as with magnetic scattering of 
neutrons. Also, it is possible to investigate non-modified bulk ferrofluids in a wide range of magnetic volume fraction of $1-20 \%$. Due to the strong polydispersity of magnetic nanoparticles dispersed in liquids, additional averaging of over the particle size is required, usually with the lognormal distribution function. E.g., SANS data obtained for $1 \%$ volume fraction FF/D-benzene and analyzed as mentioned above indicated the penetration of the solvent into the surfactant shell [54].

Aggregates formed due to particle interactions produce important changes in the scattering curves at small $q$-values (structure-factor effect). For sufficiently large $q$-values, where such effect is small or negligible, the model fitting can be made, e.g. for FF based on MEK (methyl-ethyl-ketone) with magnetite covered by double layer OA+DBS [49]. In SANS the interaction effects start to be significant at magnetic particle volume fraction $\varphi_{m}>6 \%$. They are a result of the competition between attraction (atomic Van der Waals and magnetic dipole-dipole interactions) and stabilizing repulsion (temperature and shell interaction). For isotropic interaction between monodisperse particles an additional structure-factor, $S(q)$, appears in the scattering intensity. Thus, if one neglects the polydispersity and takes into account that the magnetic scattering is isotropic in nonmagnetized magnetic fluids, the scattering intensity may be approximated as $I(q) \approx N F_{N}^{2}(q) S_{N}(q)+(2 / 3) N F_{M}^{2}(q) S_{M}(q)$, where $S_{N}(q)$ and $S_{M}(q)$ are effective structure-factors corresponding to the nuclear $\left(\mathrm{F}_{\mathrm{N}}\right)$ and magnetic $\left(\mathrm{F}_{\mathrm{M}}\right)$ scattering contributions, respectively. In the case of double layer sterically stabilized weakly polar organic FFs (e.g. FF/H-pentanol), the attractive component of the interaction potential is fully screened. It was shown [49] that the simplest model of the hard-sphere interaction in terms of the Vrij formalism [55] for polydisperse systems could be applied only for samples with $\varphi_{m}<6 \%$. The results of the fitting procedure indicate a strong interpenetration of the sublayers.

For typical SANS curves for aqueous magnetic fluids [49] with a small volume fraction of magnetite in normal water carrier, the contribution of the magnetic scattering can be neglected. The linear behavior of the scattering in the double logarithm scale at small $q$-values corresponds to the power law $S_{N}(q) \sim q^{-\alpha}$, where exponent $\alpha$ determines the type of clusters. The case $\alpha=-1$ can be related to the elongated aggregates, while the case $1<\alpha<3$ corresponds to the branched fractal clusters of the mass fractal dimension $D=\alpha$. Drop-like aggregates formed as the result of bulk condensation of particles under the action of a magnetic field [56] lead to more than one order of magnitude increase of viscosity.

\section{Magnetorheological fluids}

Magnetorheological (MR) fluids [7], foams and elastomers comprise a class of smart materials whose rheological properties may be controlled by the application of an external magnetic field [6, 8]. Multidisciplinary researches on MR fluids increased considerably after 1990 due to promising applications coming up on the marketplace. The ability to control by a moderate magnetic field (magnetic induction B less than 1T) the force or torque transmission in actuators, dampers and robotics components, is a consequence of the attractive magnetorheological behavior of MR fluids. The basic phenomena are related to reversible structural changes induced by an applied magnetic field, which give rise to an effective increase of viscosity by several orders of magnitude in a fraction of msec.

Composition and synthesis. The composition of MR fluids, is similar to that of ferrofluids: a high concentration of magnetizable particles dispersed in a non-magnetic medium with fluid properties. Significant differences in particle size and composition however result in distinct rheological behavior in applied magnetic field. In particular, MR fluid particle sizes typically range from 1-20 $\mu \mathrm{m}$, about three orders of magnitude larger than colloidal ferrofluid particles. The larger MR fluid particles allow for stable, highly magnetizable materials and reversible particle aggregation. Typical micron-sized MR particles consist of hundreds of magnetic domains and are much larger than subdomain nanoparticles with permanent dipole moment in ferrofluids. Induced dipole moment in the presence of a field causes interparticle attraction. Maximum interparticle attraction and thus 
maximum magnetorheological effect is increased by choosing a particle material with high saturation magnetization. Iron has the highest saturation magnetization of known elements, 2.1 Tesla. Iron particles with spherical shape obtained by the thermal decomposition of iron pentacarbonyl are commonly used. Alloys of iron and cobalt are known to have slightly higher saturation magnetization (up to 2.4 Tesla) and have also been used in MR fluids. Typical particle volume fractions are between 0.1 and 0.5 . Carrier liquids are typically chosen based upon their rheological and tribological properties and on their temperature stability. Typically, petroleum based oils, silicone, mineral oils, polyesters, polyethers, water, synthetic hydrocarbon oils and others are used. Additives are used to provide additional lubricating properties, as well as additives that inhibit sedimentation and agglomeration. Sedimentation is typically controlled by the use of thixotropic agents and surfactants such as xantham gum, silica gel, stearates and carboxylic acids [8]. The significant difference between iron particle density $\left(\rho \approx 7.5 \mathrm{~g} / \mathrm{cm}^{3}\right)$ and carrier fluid density ( $\rho \approx 1 \mathrm{~g} / \mathrm{cm}^{3}$ ) makes MR fluids susceptible to long-term separation.

Particle sedimentation/aggregation and suspension stability. Sedimentation stability refers to gravitational stability and ensures that the particles do not settle over time, while agglomerative stability prevents the particles from sticking together in the absence of the field.

In order to improve the stability of MR fluids against sedimentation various solutions were proposed concerning their composition and the characteristic size of the magnetic component [57].

Beside the usual composition MR fluids, the two phases can be two immiscible fluids, one of them being a ferrofluid. The application of the magnetic field will change the shape and size of the droplets of the dispersed phase (e.g. a ferrofluid) which will modify the rheology [58].

Colloidal MR fluids were synthesized using ferrite-based particles of the order of $30 \mathrm{~nm}$ in diameter [59] coated with long chain molecules. These fluids, which are very similar to ferrofluids, are reported to have excellent stability and abrasion properties. They, however, exhibit an order of magnitude less yield stress than the usual iron-based MR fluids resulting from inferior magnetic properties of ferrite and the predominance of thermal particle forces.

Bidisperse MR fluids with nanosize (non-magnetic) filler particles were proposed [60] in order to circumvent the problem of sedimentation and also to increase the magnetic field induced yield stress. In a recent work bidisperse MR fluids having nanometer $(30 \mathrm{~nm})$ and micrometer $(30 \mu \mathrm{m})$ size iron particles [61] were investigated. The ratio between the kind of powders in the composition, keeping constant the mass concentration of iron particles $(60 \% \mathrm{wt})$, influence the rheological behavior and settling properties of the MR fluid.

Water based MR fluids with longterm stability were prepared [62] by adding of soluble polymers that modify the viscosity of the carrier and adsorb on the particles, giving rise to strong hydrophilic and steric repulsion between micrometric magnetite particles.

The most promising type of MR fluid uses a ferrofluid as carrier liquid and micrometer size iron particles dispersed in the magnetizable liquid matrix [63], resulting in an extremely bidisperse MR fluid. The increase of yield stress is due to the increased force between two iron particles mediated by the carrier fluid with non-zero magnetic susceptibility. Also, sedimentation is prevented by magnetic interactions between nanosized permanent dipoles and multi-domain ferromagnetic particles, resulting in a local alignment and network formation of nanodipoles between micrometer size iron particles [6]. Nanosized particles addition to MR fluids with micrometer size ferro- or ferromagnetic particles is an alternative strategy to improve the stability of these suspensions. The sedimentation behavior of extremely bimodal suspensions, with micrometer $(1450 \mathrm{~nm})$ and nanometer $(8 \mathrm{~nm})$ size magnetite particles was examined recently [64]. Magnetic nanoparticles greatly reduce gravitational settling by increasing the viscosity and density of the medium and by the so called "halo" structure formation, as a consequence of gathering magnetic nanoparticles around the large micrometer range particles due to magnetic and van der Waals attraction.

A new type of magnetorheological fluid was developed [65] based on a carbon nanotube and magnetite (CNT/Fe3O4) nanocomposite. The MR fluid contains CNTs covered with a layer of soft magnetic magnetite nanoparticles. Due to very high length to diameter ratio of the magnetic 
component a three-dimensional network is formed which prevents settling. A significant further advance in improving the stability and increasing the yield stress of MR fluids is the use of magnetic fibers instead of spherical ferromagnetic particles [66]. Cobalt wires and iron filaments (approx. $60 \mu \mathrm{m}$ in length and 4-16 $\mu \mathrm{m}$ in width) were synthesized and dispersed in silicon oil. The MR properties of suspensions of magnetic fibers, in particular the Bingham yield stress is about three times greater compared to that measured for similar suspensions of spherical particles.

A special category of MR composites are the magnetic gels and elastomers [67, 68], which practically are not affected by sedimentation. Polymer gels through crosslinking form networks to promote particle stability. Depending on the level of crosslinking, the resulting material can be a liquid (gel) or solid. Controlling the degree of crosslinking is achieved by controlling reaction conditions. The polymerization can occur before or after the magnetic particles have been added. Improved stability can be achieved by forming the polymeric gel in the presence of the magnetic particles. The shape of magnetic gels and elastomers may be tuned by magnetic field- magnetic particles interactions. Giant deformational effect, tuneable elastic modulus, non-homogeneous deformation and quick response to magnetic field open new opportunities for using such materials for various applications. Smart hydrogel membranes were obtained starting with magnetic polystyrene latex prepared using a ferrofluid [69]. Magnetically ordered nanochannels are formed which can act as "on-off "switches or "permeability valves" in response to external stimuli.

Field induced particle interactions and structure formation. Flow properties. In case of MR fluids particle interactions when magnetic field is applied are very strong and conduct to formation of thick columns of particles, which radically change the flow behavior [6]. Consider an isolated particle of relative magnetic permeability $\mu_{p}$ in a liquid with relative magnetic permeability $\mu_{f}$, under the action of an external magnetic field $\mathrm{H}_{0}$. The particle will acquire a magnetic moment $\boldsymbol{m}=4 \pi \mu_{0} \mu_{f} \beta a^{3} \boldsymbol{H}_{0}$, where $a$ is the radius of particle, $\mu_{0}$ the permeability of vacuum and $\beta=\left(\mu_{p}-\mu_{f}\right) /($ $\mu_{p}+2 \mu_{f}$ ). The expression of $\boldsymbol{m}$ includes most of the situations described above. E.g., when the relative permeability of the carrier is greater than that of the particle, we have the case of "inverse" ferrofluid. Taking into account the interaction energy between two dipoles of moment $m$, the nondimensional interaction energy of two dipoles in repulsive configuration gives

$\lambda_{\text {int }}{ }^{M R}=\pi \mu_{0} \mu_{f} \beta a^{3} \boldsymbol{H}_{0}{ }^{2} /(2 k T)$, expression which is quite different from $\lambda_{\text {int }}=\mu_{0} m^{2} /\left(2 \pi k_{B} T d_{m}{ }^{3}\right)$ valid for magnetic fluids. Indeed $\lambda_{\text {int }}{ }^{M R} \sim \boldsymbol{H}_{0}{ }^{2}$ in case of MR fluids and $\lambda_{\text {int }}{ }^{M R}$ attains 1 already at very low field values, $\mathrm{H}=127 \mathrm{~A} / \mathrm{m}$ for $2 a=1 \mu \mathrm{m}$. Usually, in case of MR fluids $\lambda_{\text {int }}{ }^{M R}{ } » 1$, i.e. already for moderate magnetic fields the magnetic forces dominate the Brownian forces which sometimes can be neglected.

The yield stress $\tau_{y}$ as a function of the magnetic induction B is the main property envisaged for applications of MR suspensions. Experimentally the static yield stress is obtained by extrapolating at zero shear rate the stress vs. logarithm of shear rate curve (there must be data until $10^{-3} \mathrm{~s}^{-1}$ ) [6]. Note, however, that the yield stress is not an enough well defined quantity and does not represent a bulk property of the fluid. The Bingham $\tau^{1 / 2}=\tau_{d}^{1 / 2}+(\eta \dot{\gamma})^{1 / 2}$ or Herschel-Bulkley $\tau=\tau_{d}+(K \dot{\gamma})^{p}$ approximation of the flow curves gives a more reliable quantity, $\tau_{d}$ - the dynamical yield stress. This stress represents the one needed to continuously separate the particles against the attractive magnetic forces in the low shear limit [6]. The maximum yield stress at high magnetic induction will be limited by the magnetic saturation and attains $50-100 \mathrm{kPa}$ for most of MR fluids, which is about an order of magnitude larger than for electrorheological (ER) fluids. The nondimensional magnetic dipole energy $\lambda_{\text {int }}$ is related to the Mason number Mn (the ratio of shear to magnetic forces) and to the Peclet number Pe (the ratio of shear forces to Brownian forces) by the relation $M n \lambda_{\text {int }}=2 P e / 3$, i.e. for a given MR fluid the viscosity will be the same for the same values of $M n$ and $\lambda_{\text {int }}$. 


\section{Summary}

The structure and properties of magnetically controllable fluids - ferrofluids and MR fluids - may be finely tuned to fulfill the requirements of a large diversity of engineering and biomedical applications by the proper choice of composition and by the synthesis procedures applied.

Acknowledgement. This work is dedicated to the memory of Dr. Doina Bica, to her outstanding contribution to magnetic fluids science. It was completed in the framework of the 2005-2009 Research programme of the Lab. of MFs - CFATR, being partly supported also by the NanoMagneFluidSeal and FeMANANOF research projects of the CEEX programme of the National Authority for Scientific Research.

\section{References}

1. R.E. Rosensweig, Ferrohydrodynamics, Cambridge Univ. Press, Cambridge (1985)344p, reprinted with slight corrections by Dover, Mineola, New York (1997)

2. M.I. Shliomis, Soviet Phys. Uspekhi (Engl. transl) 17, 34 (1974)

3. E. Blums, A. Cebers, M.M. Mayorov, Magnetic fluids, Walter de Gruyters, Berlin (1997)

4. S. Odenbach, Ferrofluids, in: K.H.J. Buschow (Ed), Handbook of Magnetic Materials, vol.16, chap.3 (2006) pp.127-208

5. I. Anton, I. De Sabata, L. Vekas, J. Magn. Magn. Mater., 85, 219 (1990)

6. G. Bossis, O. Volkova, S. Lacis, A. Meunier, Magnetorheology: Fluids, Structures and Rheology, in: S. Odenbach (Ed) Ferrofluids. Magnetically controllable fluids and their applications (Lecture Notes in Physics; 594; Springer-Verlag 2002) pp. 202-230

7. Z. P. Shulman, V. I. Kordonsky, E. A. Zaltsgendler, I. V. Prokhorov, B. M. Kushid, S.A. Demchuk, Int. J. Multiphase Flow, 12, 935(1986)

8. J. D. Carlson, M. R. Jolly, Mechatronics, 10, 555(2000)

9. S. W. Charles, in: S. Odenbach (Ed) Ferrofluids. Magnetically controllable fluids and their applications (Lecture Notes in Physics; 594; Springer-Verlag 2002) p.3-18

10. S.E. Khalafalla, G.W. Reimers, US Patent 3764540(1973)

11. S. W. Charles, Romanian Reports in Physics, 47, 249(1995)

12. D. Bica, Romanian Reports in Physics, 47, 265(1995)

13. D. Bica, RO Patent 90078 (1985)

14. G. D. Moeser, K. A. Roach, W. H. Green, P. E. Laibinis, T.A. Hatton, Ind. Eng. Chem. Res., 41, 4739(2002)

15. M.-P. Pileni, Langmuir, 13,639(1997)

16. M.-P. Pileni, N. Feltin, N. Moumen, in: Scientific and Clinical Applications of Magnetic Carriers (Eds. U. Häfeli, W. Schütt, M. Zborowski, Plenum Press (New York, London, 1997) pp.117-133

17. U. Jeong, X. Teng, Y. Wang, H. Yang, Y. Xia, Adv. Mater.,19, 33(2007)

18. T. Hyeon, Chem. Commun., 927(2003)

19. T. Hyeon, S. S. Lee, J. Park, Y. Chung and H. B. Na, J. Am. Chem. Soc.,123, 12798(2001)

20. S. Sun, H. Zeng, J. Am. Chem. Soc.124, 8204 (2002) 
21. S. Sun, H. Zeng, D. B. Robinson, S. Raoux, P. M. Rice, S. X. Wang, G. Li, J. Am. Chem. Soc.126, 273(2004)

22. S. Neveu, A. Bee, M. Robineau, D. Talbot, J.Colloid \& Interface Sci. 255, 293(2002)

23. H. Bönnemann, R. A. Brand, W. Brijoux, H. W. Hofstadt, M. Frerichs, V. Kempter, W. MausFriedrichs, N. Matoussevitch, K. S. Nagabhushana, F. Voigts, and V. Caps, Appl. Organomet. Chem. 19, 790 (2005)

24. F. Dumitrache, I. Morjan, R. Alexandrescu, V. Ciupina, G. Prodan, I.Voicu, C. Fleaca, I. Albu, M. Savoiu, I. Sandu, E. Popovici, I. Soare, Appl.Surf. Sci. 247, 25 (2005)

25. E. Popovici, F. Dumitrache, I. Morjan, R. Alexandrescu,V. Ciupina, G. Prodan, L. Vékás, D. Bica, O. Marinica, E. Vasile, Appl. Surf. Sci. 254(4)1048(2007)

26. R. W. Chantrell, A. Bradbury, J. Popplewell, S.W. Charles, J. Phys. D13, L119 (1980)

27. D. Bica, L. Vékás, Magnitnaia Ghidrodinamika (Magnetohydrodynamics) 30 (3)194 (1994)

28. D. Bica, L. Vékás, M. Rasa, J. Magn. Magn. Mater. 252, 10(2002)

29. D. Bica, L. Vékás, M. V. Avdeev, M. Balasoiu, O. Marinica, F. D. Stoian, D. Susan-Resiga, G. Török, L. Rosta, Progr. Colloid \& Polymer Sci., 125 1(2004)

30. L. Vékás, D. Bica, O. Marinica, M. Rasa, V. Socoliuc, F. D. Stoian, J. Magn. Magn. Mater., 289, 50(2005)

31. L. Vékás, D. Bica, M. V. Avdeev, China Particuology, 5, 43(2007)

32. I. Borbáth, Z. Kacsó, L. Dávid, I. Potencz, D. Bica, O. Marinica, L.Vékás, In: Convergence of micro-nano-biotechnologies, Series Micro- and Nanoengineering, Romanian Academy Publ. House (Bucharest, 2006) p. 200

33. S. Behrens, H. Bönemann, N. Matoussevitch, E. Dinjus, H. Modrow, N. Palina, M. Frerichs, V. Kempter, W. Maus-Friedrichs, A. Heinemann, M. Kammel, A. Wiedenmann, L. Pop, S. Odenbach, E. Uhlmann, N. Bayat, J. Hesselbach, J. M. Guldbakke, Z. Phys. Chem. 220, 3 (2006)

34. S. Behrens, H. Bönemann, N. Matoussevitch, A. Gorschinski, E. Dinjus, W. Habicht, J. Bolle, S. Zinoveva, N. Palina, J. Hormes, H. Modrow, S. Bahr, V. Kempter, J. Phys: Condens. Matter, 18, S2543 (2006)

35. R. Massart, IEEE Trans. Magn., MAG-17, 1247(1981)

36. R. Massart, E. Dubois, V. Cabuil, E. Hasmonay, J.Magn.Magn.Mater., 149, 1(1995)

37. R. Massart, in: Magnetic Fluids and Applications Handbook (Eds. B. Berkovski, V. Bashtovoy) Begell House Inc. (1996) (New York, Wallingford (UK)) 24

38. J. Shimoiizaka, K. Nakatsuka, T. Fujita, A. Kounosu, IEEE Trans. Magn., MAG-16, 368(1980)

39. S. E. Khalafalla, G.W. Reimers, IEEE Trans. Magn. MAG-16, 178(1980)

40. A. Wooding, M. Kilner, D. B. Lambrick, J. Colloid \& Interface Sci., 144, 236 (1991)

41. A. Wooding, M. Kilner, D.B. Lambrick, J. Colloid \& Interface Sci., 149, 98(1992)

42. L. Shen, P. E. Laibinis, T. A. Hatton, Langmuir, 15, 447(1999)

43. G. D. Moeser, W. H. Green, P. E. Laibinis, P. Linse, T. A. Hatton, Langmuir, 20, 5223(2004)

44. M. Lattuada, T. A. Hatton, Langmuir 23, 2158(2007) 
45. E. Illés, E. Tombácz J. Colloid \& Interface Sci. 295, 115(2006)

46. Q. A. Pankhurst, J. Connolly, S.K. Jones, J. Dobson, J. Phys. D. Appl. Phys. 36, R167 (2003)

47. T. Neuberger, B. Schöp, H. Hofmann, M. Hofmann, B. von Rechenberg, J. Magn. Magn. Mater., 293, 483(2005)

48. V. Socoliuc, D. Bica, Progr. Colloid Polym.Sci.,117, 131(2001)

49. M. V. Avdeev, V. L. Aksenov, M. Balasoiu, V. Garamus, A. Schreyer, G. Torok, D. Hasegan, L. Rosta, D. Bica, L. Vékás, J. Colloid \& Interface Sci., 295, 100(2006)

50. R. Jurgons, C. Seliger, A. Hilpert, L. Trahms, S. Odenbach,C. Alexiou, J. Phys.: Condens. Matter 18, S2893 (2006)

51. D. Bica, L.Vékás, M. V. Avdeev, O. Marinica, M. Balasoiu, V. M. Garamus, J. Magn. Magn. Mater. 311, 17(2007)

52. E. Tombácz, D. Bica, A. Hajdú, E. Illés, A. Majzik, L. Vékás, J. Phys.: Condens. Matter (accepted; to appear in 2008)

53. M. V. Avdeev, J. Appl. Cryst., 40, 56(2007)

54. M. V. Avdeev, M. Balasoiu, Gy. Torok, D. Bica, L. Rosta, V. Aksenov, L. Vékás, J. Magn. Magn. Mater. 252, 86(2002)

55. D. Frenkel, R. J. Vos, C. G. de Kruif, A. Vrij, J. Chem. Phys., 84, 4625(1986)

56. A. Y. Zubarev, L.Y. Iskakova, Physica A 343, 65(2004)

57. F. D. Goncalves, J.-H. Koo, M. Ahmadian, The Shock and Vibration Digest 38,203(2006)

58. A. P. Gast, C.F. Zukovski, Adv.Coll.Int. Sci., 30, 53(1989)

59. C. H. Kormann, M. Laun, H.J. Richter, Int. J. Mod. Phys., B 10, 3167 (1996)

60. R. T. Foister, US Patent 5667715 (1997)

61. N. M. Wereley, A. Chaudhuri, J. -H. Yoo, S. John, S. Kotha, A. Suggs, R. Radhakrishnan, B. J. Love and T. S. Sudarshan, Journal of Intelligent Material Systems and Structures, 17,393( 2006)

62. J. L. Viota, J. de Vicente, J. D. G. Duran, A.V. Delgado, J. Colloid \& Inteface Sci. 284, 527 (2005)

63. J. M. Ginder, L.D. Elie, L. C. Davies, US Patent 5549837 (1996)

64. J. L. Viota, F. Gonzalez-Caballero, J. D. G. Duran, A. V. Delgado, J.Colloid \& Interface Sci. 309, 135(2007)

65. H. Pu, F. Jiang, Nanotechnology, 16, 1486(2005)

66. M. T. Lopez-Lopez, G. Vertelov, G. Bossis, P. Kuzhir, J.D.G. Duran, J. Mater. Chem. 17, $3839(2007)$

67. M. Zrinyi, L. Barsi, A. Büki, J. Chem. Phys.,104(20)8750(1996)

68. Zs. Varga, G. Filipcsei, M. Zrinyi, Polymer, 46, 7779(2005)

69. I. Csetneki, G. Filipcsei, M. Zrinyi, Macromolecules, 39, 1938(2006) 